\title{
The effect of the spin-orbit geometric phase on the spectrum of Aharonov-Bohm oscillations in a semiconductor mesoscopic ring
}

\author{
A. G. Mal'shukov and V. V. Shlyapin \\ Institute of Spectroscopy, Russian Academy of Science, 142092 Troitsk, Moscow oblast, Russia \\ K. A. Chao \\ Department of Theoretical Physics, Lund University, S-223 62 Lund, Sweden
}

(July 8, 2021)

\begin{abstract}
Taking into account the spin precession caused by the spinorbit splitting of the conduction band in semiconductor quantum wells, we have calculated the Fourier spectra of conductance and state-density correlators in a $2 \mathrm{D}$ ring, in order to investigate the structure of the main peak corresponding to Aharonov-Bohm oscillations. In narrow rings the peak structure is determined by the competition between the spin-orbit and the Zeeman couplings. The latter leads to a peak broadening, and produces the peak splitting in the state-density Fourier spectrum. We have found an oscillation of the peak intensity as a function of the spin-orbit coupling constant, and this effect of the quantum interference caused by the spin geometric phase is destroyed with increasing Zeeman coupling.
\end{abstract}

73.23.-b, 03.65.Bz, 71.70.Ej

The spin-orbit interaction (SOI) gives rise to a geometric phase in the quantum amplitude of a particle propagating along a closed trajectory. In ideal $1 \mathrm{D}$ rings this can lead to quantum oscillations of transport parameters similar to the Aharonov-Bohm effect [1,2]. In disordered conductors the interference between two time reversed paths produces the oscillation of mean conductance analogous to the Altshuler-Aronov-Spivak effect. Such oscillation in 1-D systems was shown by Meir, Gefen and Entin-Wohlman [3], and in systems of higher dimensions by Mathur and Stone 4 . Besides, SOI also modifies the shape of the Aharonov-Bohm and Altshuler-AronovSpivak oscillations. For a disordered material, the mean conductance is obtained as an ensemble average over a large number of measurements on different samples. One can try to detect the quantum effects associated to the spin-orbit phase by measuring the oscillations of mean conductance when the spin-orbit coupling strength or the external magnetic field is varied. To our knowledge, such experiments have not yet established any evidence of the spin-orbit geometric phase.

For a disordered material, if one takes the Fourier transform of the mean conductance $\langle g(B)\rangle$ as a function of the external magnetic field $B$, the spectrum is dominated by the Altshuler-Aronov-Spivak oscillations which is periodic in magnetic flux with a period $h c / 2 e$. On the other hand, if one takes first the Fourier transform $g(\nu)$ of a measured conductance $g(B)$, and then performs an ensemble average $\langle|g(\nu)|\rangle$ of the Fourier amplitude, one would expect that the so-derived spectrum will exhibit a main peak corresponding to the Aharonov-Bohm oscillations with a period $h c / e$ in magnetic flux [5]. Consequently, the average of Fourier amplitude, $\langle|g(\nu)|\rangle$ represents correlations of conductances measured at different magnetic fields. The dependence of these correlations on the SOI can then manifest itself in the shape of the mean peak. In a recent experiment [6] on mesoscopic rings made from a AlSb/InAs quantum well structure, the data were analyzed in this way for the first time, and a split of the main peak in the measured spectrum $\langle|g(\nu)|\rangle$ was observed. The authors of Ref. [6] have conjectured that the observed splitting is due to the strong Rashba SOI in a doped AlSb/InAs quantum well.

While the experimental results in Ref. [6] remain to be explained, in our opinion, the fundamental question needed to be answered is how the Fourier spectrum of the conductance correlations, particularly the shape of the main Fourier peak, is influenced by the interplay between the spin-orbit phase and the external magnetic field. This is the aim of the present Letter. The more suitable starting point for such a theoretical analysis is

$$
\left\langle|g(\nu)|^{2}\right\rangle=\iint_{-B_{0}}^{B_{0}} d B d B^{\prime} e^{i \nu\left(B-B^{\prime}\right)}\left\langle g(B) g\left(B^{\prime}\right)\right\rangle,
$$

where the interval between $-B_{0}$ and $B_{0}$ covers the region in which $g(B)$ is measured. This interval is much larger than both the period of the Aharonov-Bohm oscillations and the magnetic field correlation range [8] of mesoscopic fluctuations. In this Letter we will calculate (11) in the diffusion regime of a disordered $2 \mathrm{D}$ semiconductor ring of width $w$ and radius $R$. Our theory explains the main physical mechanisms which determine the shape of the Fourier spectrum. For the state-density correlator which partly contributes to (1) we predict a split of the main Fourier peak when effect of the Zeeman interaction is not completely suppressed by SOI and mesoscopic fluctuations. After we point out that this same feature appears in an ideal ballistic ring, we conjecture later that our theory also provides the physical origin of main peak splitting observed in a chaotic ballistic ring [6].

Since it is the Rashba [9] term rather than the Dresselhaus [10] term which gives the major contribution to the SOI in InAs based QW [11], for simplicity in this Letter we will neglect the Dresselhaus term. We will 
use the standard perturbation theory which was applied previously [8] to analyze the conductance correlations. In the framework of this theory the correlator in (11) is expressed via two-particle propagators (Cooperons and diffusons) where one of the particles propagates at the magnetic field $B$ and the other at $B^{\prime}$. If one neglects the Zeeman interaction with the external magnetic field, the Cooperon propagator is a function of $B+B^{\prime}$, while the diffuson depends only on $\Delta B=B-B^{\prime}$. Hence, in the vicinity of the main peak at $\nu=2 \pi^{2} R^{2} / \Phi_{0}$, where $\Phi_{0}=h c / e$ is the flux quantum, the major contribution to (11) is given by diffusons. If the Zeeman interaction is taken into account, the Cooperon propagator is no longer a function of $B+B^{\prime}$ alone. Nevertheless, Cooperon's contribution is relatively small if the Zeeman energy $g \mu_{B} \Phi_{0} / \pi R^{2}$ is much less than the Thouless energy $E_{T}=D \hbar / R^{2}$. Therefore, in the integrand of (11) we will retain only the part of the correlator associated to diffusons.

Each of the diffusons is a component of a matrix $\mathbf{D}\left(\mathbf{r}, \mathbf{r}^{\prime}\right)$ with four spin indices representing the spin states of an electron and a hole. Following Ref. [8] one can show that the correlator in the integrand of (1) is proportional to

$$
\begin{aligned}
& \int d^{2} r d^{2} r^{\prime}\left\{\operatorname{Re}\left(\operatorname{Tr}\left[\mathbf{D}\left(\mathbf{r}, \mathbf{r}^{\prime}\right) \mathbf{D}\left(\mathbf{r}^{\prime}, \mathbf{r}\right)\right]\right)\right. \\
& \left.+2 \operatorname{Tr}\left[\mathbf{D}\left(\mathbf{r}, \mathbf{r}^{\prime}\right) \mathbf{D}^{\dagger}\left(\mathbf{r}, \mathbf{r}^{\prime}\right)\right]\right\}
\end{aligned}
$$

In the above equation the trace is taken separately over the electron and hole spin indices. It is convenient to express $\mathbf{D}$ in the representation of the total spin $\mathbf{S}$ of the electron-hole two-particle system [12]. In this representation $\mathbf{D}$ is a $4 \times 4$ matrix with components $D_{m n}$. The indices $m$ and $n$ can have the values -1,0,1 (for the $z$ component of the triplet) and $s$ (for the singlet).

In $\mathrm{A}_{3} \mathrm{~B}_{5}$ semiconductors the spin-orbit coupling has the form $H_{s o}=\mathbf{h}_{\mathbf{k}} \cdot \mathbf{s}$ for an electron having spin $\mathbf{s}$ and quasi-momentum k. This SOI and the Zeeman interaction determine the spin dependence of $\mathbf{D}$, which can be written as 13]

$$
\begin{aligned}
& \tau\left\langle\left(-i \mathbf{v} \cdot \nabla+\frac{e}{c} \mathbf{v} \cdot \Delta \mathbf{A}+\mathbf{h}_{\mathbf{k}} \cdot \mathbf{S}\right)^{2}\right\rangle_{a n g} \mathbf{D}\left(\mathbf{r}, \mathbf{r}^{\prime}\right) \\
& +\mathbf{Z D}\left(\mathbf{r}, \mathbf{r}^{\prime}\right)+\frac{1}{\tau_{\varphi}} \mathbf{D}\left(\mathbf{r}, \mathbf{r}^{\prime}\right)=\delta\left(\mathbf{r}-\mathbf{r}^{\prime}\right),
\end{aligned}
$$

where $\tau_{\varphi}$ is the elastic mean free scattering time, $\tau$ the phase breaking time, and the notation $\langle\cdots\rangle_{a n g}$ is an angular average over the Fermi surface. The term $\mathbf{Z D}\left(\mathbf{r}, \mathbf{r}^{\prime}\right)$ in the above equation is due to the Zeeman interaction, and the nonzero components of $\mathbf{Z}$ are

$$
\begin{aligned}
& Z_{0 s}=Z_{s 0}=\frac{i g \mu_{B}}{2}\left(B-B^{\prime}\right) \\
& Z_{11}=-Z_{-1-1}=\frac{i g \mu_{B}}{2}\left(B+B^{\prime}\right) .
\end{aligned}
$$

We see that if $B \neq B^{\prime}$ the matrix $\mathbf{Z}$ contains components which mix the singlet part and the triplet part of the diffuson.
We choose the gauge such that for the field difference $\Delta B$ the vector potential is $\Delta \mathbf{A}=\Delta B r \mathbf{t} / 2$, where $\mathbf{t}$ is a unit vector tangential to the ring. Since $H_{s o}=\alpha\left(k_{x} \mathbf{s}_{\mathbf{y}^{-}}\right.$ $k_{y} \mathbf{s}_{\mathbf{x}}$ ) when the Rashba term dominates, the boundary conditions at the inner and the outer radii of the ring are

$$
-i \frac{\partial}{\partial r} \mathbf{D}\left(\mathbf{r}, \mathbf{r}^{\prime}\right)-\frac{\alpha m^{*}}{\hbar}(\mathbf{t} \cdot \mathbf{S}) \mathbf{D}\left(\mathbf{r}, \mathbf{r}^{\prime}\right)=0 .
$$

In a narrow ring with the width $w$ much less than the radius $R, \mathbf{D}\left(\mathbf{r}, \mathbf{r}^{\prime}\right)$ varies slowly across the annulus. If the elastic mean free path $l$ is much shorter than $w$, such slow variation can be treated perturbatively in the diffusion approximation [14]. Using the boundary conditions (5), after averaging over $r$, (3) is reduced to an effectively $1 \mathrm{D}$ equation, where $\mathbf{D}$ depends only on the azimuthal angles $\varphi, \varphi^{\prime}$. This function can be expressed in the form

$$
\mathbf{D}\left(\varphi, \varphi^{\prime}\right)=e^{i \mathbf{S}_{z}\left(\varphi^{\prime}-\varphi\right)} \sum_{n} \mathbf{M}_{n}\left(\varphi^{\prime}\right) e^{i \varphi n} .
$$

Making use of the rotation properties of the spin operator

$$
\exp \left[i \mathbf{S}_{z}\left(\varphi-\varphi^{\prime}\right)\right](\mathbf{S} \cdot \mathbf{n}) \exp \left[i \mathbf{S}_{z}\left(\varphi^{\prime}-\varphi\right)\right]=\left(\mathbf{S} \cdot \mathbf{n}^{\prime}\right),
$$

where $n_{x}^{\prime}=\cos \varphi^{\prime}$ and $n_{y}^{\prime}=\sin \varphi^{\prime}$, we arrive at a set of $4 \times 4$ algebraic equations for the components of the matrices $\mathbf{M}_{n}$. From these equations one can derive the following subset of equations which contains only the -1,0,1 components of the matrices $\mathbf{M}_{n}$

$[n-\Delta \phi-\zeta(\mathbf{S} \cdot \mathbf{N})]^{2} \mathbf{M}_{n}+\left[a+\rho^{2} M_{0}\left(1-\mathbf{S}_{z}^{2}\right)\right](\Delta \phi)^{2} \mathbf{M}_{n}$ $+i \rho \mathbf{S}_{z}\left(\phi+\phi^{\prime}\right) \mathbf{M}_{n}+\frac{1}{\tau_{\varphi} E_{T}} \mathbf{M}_{n}=\mathbf{1}$,

where $a=w^{2} / 4 R^{2}, \quad M_{0}=\left[(n-\Delta \phi)^{2}+a(\Delta \phi)^{2}+1 / \tau_{\varphi} E_{T}\right]^{-1}$, $\zeta^{2}=1+\left(\operatorname{R\alpha m}^{*} / \hbar\right)^{2}$, and $\rho=g m^{*} / k_{f} l m$. $\mathbf{N}$ is a unit vector with $N_{x}=n_{x}^{\prime}\left(1-\zeta^{-2}\right)^{1 / 2}, N_{y}=n_{y}^{\prime}\left(1-\zeta^{-2}\right)^{1 / 2}$ and $N_{z}=1 / \zeta$.

The three dimensionless parameters $a, \zeta$ and $\rho$ determine the shape of the main peak in $\left\langle|g(\nu)|^{2}\right\rangle$ given by (1). $a$ describes the dephasing due to the penetration of the magnetic flux $\Delta \phi=\Delta B \pi R^{2} / \Phi_{0}$ into the annulus of the ring. We should remind the reader that to calculate $a$ we have assumed diffusive propagation of particles in the radial direction. However, it is reasonable to believe that the dependence on flux of the form $a(\Delta \phi)^{2}$, as appearing in (7), is also valid for rings with ballistic transport along radial direction. In this case $a$ can be considered as a phenomenological parameter. The spin-orbit coupling constant $\zeta$ gives the spin-phase winding number after a particle has traversed a closed path along the ring. The parameter $\rho$ is related to the Zeeman interaction. It determines the amount of mixing between the triplet and the singlet components of the diffusion propagator. This mixing appears in (7) in the form $\rho^{2} M_{0}\left(1-\mathbf{S}_{z}^{2}\right)$. Hence, although $\rho$ is small, the effect of mixing is enhanced by the resonance of the singlet diffusion mode and the $S_{z}=0$ component of the triplet. However, with stronger spinorbit coupling the system is driven out of the resonance due to the term $\zeta(\mathbf{S} \cdot \mathbf{N})$ in (7). 


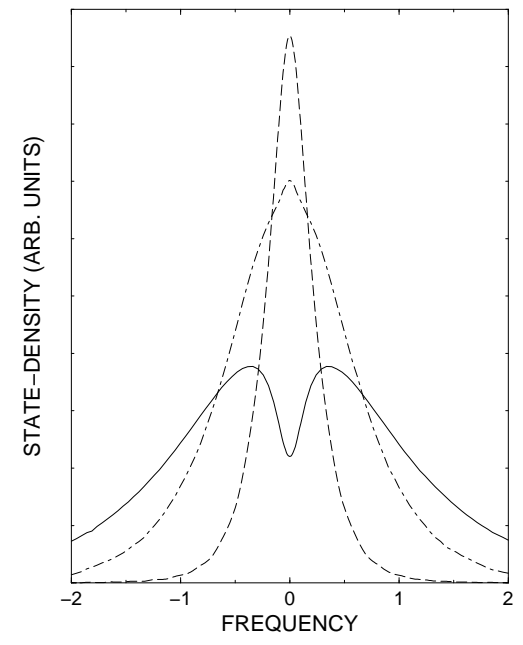

FIG. 1. Fourier spectra of state-density for various Zeeman coupling $\rho$ and SOI strength $\zeta: \rho=0.006, \zeta=1$ (solid curve); $\rho=0.0033, \zeta=1$ (dot-dashed curve); $\rho=0.006, \zeta=2.5$ (dahsed curve). The frequency is defined as $\omega=2 \pi-\nu \Phi_{0} / \pi R^{2}$.

After substituting (6) into (2) and carrying out the integration, we need to perform a numerical summation over $n$ in order to obtain the conductance fluctuations and the state-density fluctuations which, according to Ref. [15, are given by the first term of (2). The summation over $n$ is from $-n_{\max }$ to $n_{\max }=50$, which gives converging results. The magnetic field $B_{0}$ is set at a value corresponding to a flux of $300 \Phi_{0}$. Within a reasonable range of material parameters our numerical results depend only weakly on the value of $B_{0}$.

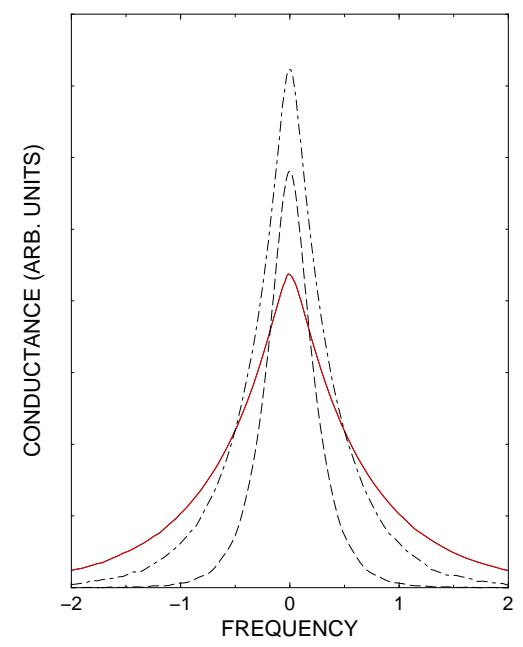

FIG. 2. Fourier spectra of conductance: $\rho=0.006, \zeta=1$ (solid curve); $\rho=0.0033, \zeta=1$ (dot-dashed curve); $\rho=0.006$, $\zeta=2.5$ (dahsed curve). The frequency is $\omega=2 \pi-\nu \Phi_{0} / \pi R^{2}$.

Figs. 1-3 were calculated with $a=2 \cdot 10^{-4}$ and $1 / \tau_{\varphi} E_{T}=$ $10^{-2}$. For the convenience of presentation, the frequency in Figs. 1 and 2 is defined as $\omega=2 \pi-\nu \Phi_{0} / \pi R^{2}$ which is dimensionless. Fig. 1 shows the main Fourier peak of the state density correlator. The splitting of the peak (solid curve) for $\rho=0.006$ and $\zeta=1$ is due to the resonance of diffusion modes. As the Zeeman coupling is reduced to $\rho=0.0033$ (dot-dashed curve), or as the resonance is detuned by increasing the SOI to $\zeta=2.5$ (dashed curve), the phenomenon of peak splitting disappears. We have also found vanishing of this splitting when the value of the parameter $a$ is enhanced, corresponding to a decrease of the magnetic field correlation range of mesoscopic fluctuations. While the splitting is seen in the state-density peak, it is absent in the diffusion coefficient spectrum. In the parameter regime considered here, the contribution to the conductance correlator from the diffusion coefficient spectrum is larger than that from the state-denstiy correlator. Consequently, there is no peak splitting in the Fourier spectrum of conductance oscillations as shown in Fig. 2.

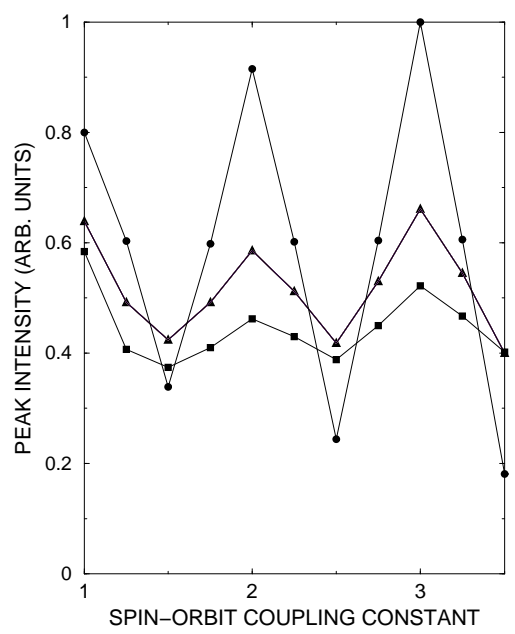

FIG. 3. The intensity of the main Fourier peak as a function of the SOI coupling: $\rho=10^{-4}$ (cicular dots); $\rho=5 \cdot 10^{-4}$ (triangles); $\rho=10^{-3}$ (squares).

Our theory predicts a quantum oscillation of the intensity of the main peak in the Fourier spectrum of conductance oscillation, as a function of the SOI strength. To demonstrate that its origin lies in the geometric phase, let us first set the Zeeman interaction $\rho=0$. In this case (7) can be easily diagonalized by choosing the spin quantization axis along $\mathbf{N}$. The eigenvalues of $\zeta \mathbf{S} \cdot \mathbf{N}$ are $\zeta$, 0 and $-\zeta$. Including the singlet state, the eigenvalue 0 is doubly degenerate. The dependence of $\left\langle|g(\nu)|^{2}\right\rangle$ in (1) from $\zeta$ can then be readily calculated, because the $\pm \zeta$ can be absorbed by a shift of $\Delta \phi$ in corresponding components of the correlator. If we ignore the small change produced by this shift in the $a(\Delta \phi)^{2}$ term, after taking trace, $\left\langle|g(\nu)|^{2}\right\rangle$ is found to be proportional to $1+\cos [(2 \pi-$ $\omega) \zeta$. At the center of the peak, $\omega=0$ and so $\left\langle|g(\nu)|^{2}\right\rangle$ oscillates with $\zeta$ as $\cos ^{2} \pi \zeta$. Since the Zeeman interaction breaks the time inversion symmetry and thus leads to an additional dephasing, the magnetic field correlation 
length is reduced and hence the dependence on the geometric phase gets weaker. As a result, the oscillating amplitude of $\left\langle|g(\nu)|^{2}\right\rangle$ decreases rapidly with increasing Zeeman interaction. The numerical result of this oscillation is shown in Fig. 3 for $\rho=10^{-4}$ (cicular dots), $\rho=5 \cdot 10^{-4}$ (triangles), and $\rho=10^{-3}$ (squares).

The splitting of the main Fourier peak has been observed in a recent experiment on AlSb/InAs/AlSb quantum well rings with radii about $1 \mu \mathrm{m}[6]$. The material parameters for these samples are $l=10^{-4} \mathrm{~cm}, g=14$, and $\alpha \simeq 10^{-9} \mathrm{eV} \mathrm{cm} \mathrm{[11],} \mathrm{which} \mathrm{give} \rho \simeq 10^{-3}$ and $\zeta \simeq 3$. With these values of $\zeta$ and $\rho$, our calculation does not yield the splitting of the main Fourier peak in both the statedensity and the conductance. However, this is not a total surprise because the samples used in Ref. [6] are very small with $l \simeq R$, which is nearly in ballistic regime instead of in diffusion regime. Furthermore, in these samples the spin precession length $\hbar^{2} / \alpha m^{*}$ is shorter than the elastic mean free path, for which our perturbative treatment of SOI is not valid.

In order to judge how relevant are our qualitative results to the above mentioned experiment, let us consider the opposite limit of an ideal 1D ring. The period of the Aharonov-Bohm (AB) oscillations changes due to the dependence of the geometric phase and dynamic spinphase on the magnetic field. We will consider first the effect of geometric phase. In the region of our interest $\rho^{\prime} \phi \ll\left(\zeta^{2}-1\right)^{1 / 2}$ with $\rho^{\prime}$ defined as $\rho^{\prime}=g m^{*} / k_{f} R m$, for an ideal ring one gets from Refs. [1] 2] the geometric phase $\theta_{g}= \pm \pi \rho^{\prime}\left(\zeta^{2}-1\right)^{-1 / 2} \phi+C$, where $C$ is a constant independent of the magnetic field, and the \pm signs refer to the two electron spin orientations. Combining the geometric phase to the $\mathrm{AB}$ phase $2 \pi \phi$, we see that SOI leads to a split of the $\mathrm{AB}$ oscillations in the transmittance of the ring into two oscillations with close frequencies. However, this frequency splitting is about two orders of magnitude less than the observed value [6]. Next, we consider the split of the $\mathrm{AB}$ oscillation frequency caused by dynamic spin phases, which depend on the magnetic field in the form $\pm \pi \delta k_{f} R$, where $\pm \delta k_{f}$ are the shifts of the Fermi wave-vector for up- and down-spin electrons. The shift $\pi \delta k_{f} R$ has its maximum value $\pi \rho^{\prime} \phi / 2$ in the absence of the SOI, and decreases with increasing SOI strength [1,2]. Even at the largest value $\pi \rho^{\prime} \phi / 2$, the corresponding split in $\mathrm{AB}$ oscillation frequency is of the order $\rho^{\prime}$ which is too small to explain the experimental value.

The general feature of the peak splitting for the ideal 1D ballistic ring is then similar to that for the diffusive $2 \mathrm{D}$ ring. Furthermore, in the $2 \mathrm{D}$ ring the amount of the $\mathrm{AB}$ oscillations splitting as seen in the state-density correlations also decreases with increasing SOI. If we use the same value for $\rho^{\prime}$ in a $1 \mathrm{D}$ ballistic ring and for $\rho$ in a $2 \mathrm{D}$ diffusive ring, which means the same strength of the Zeeman coupling in both systems, in the absence of SOI, the peak splitting in state density correlations is larger than that in the transmittance of a $1 \mathrm{D}$ ring. This is due to the longer paths traversed by a diffusing particle in its random walk along a ring, and so acquiring a larger dynamic phase. We have reached the conclusion that the two quite distinct limiting cases have led to the same qualitative picture. Consequently, we conjecture that the peak splitting observed in near ballistic 2D samples [6] is due to the Zeeman interaction which is not completely suppressed by the SOI and the mesoscopic fluctuations.

We acknowledge the support of the Royal Swedish Academy of Science under the Research Cooperation Program between Sweden and the former Soviet Union, Grant No. 12527, and of the Russian Foundation for Basic Research under Grant No. 97-02-17324.

[1] A. G. Aronov and Y. B. Lyanda-Geller, Phys. Rev. Lett. 70, 343 (1993).

[2] Y. S. Yi, T. Z. Qian, and Z. B. Su, Phys. Rev. B 55, 10631 (1997).

[3] Y. Meir, Y. Gefen, and O. Entin-Wohlman, Phys. Rev. Lett. 63, 798 (1989).

[4] H. Mathur and A. D. Stone, Phys. Rev. Lett. 68, 2964 (1992).

[5] R. Washburn and R. Webb, Rep. Prog. Phys. 55, 1311 (1992)

[6] A. F. Morpurgo, J. P. Heida, T. M. Klapwijk, B. J. Wees, and G. Borghs, Phys. Rev. Lett. 80, 1050 (1998).

[7] In fact, experimentally measured was the magnetoresistance. However, we are interested in the metallic regime. Hence, the statistical properties of conductance and resistance are the same.

[8] B. L. Al'tshuler and D. E. Khmel'nitskii, JETP Letters 42, 359 (1985); P. A. Lee, A. D. Stone, and H. Fukuyama, Phys. Rev. B 35, 1039 (1987).

[9] Yu. L. Bychkov and E. I. Rashba, J. Phys. C 17, 6093 (1984).

[10] G. Dresselhaus, Phys. Rev. 100, 580 (1955).

[11] J. Nitta, T. Akazaki, H. Takayanagi, and T. Enoki, Phys. Rev. Lett. 78, 1335 (1997).

[12] B. L. Altshuler and A. G. Aronov, in Electron-Electron Interactions in Disordered Systems, edited by A. L. Efros and M. Pollak (North-Holland, Amsterdam, 1985).

[13] S. V. Iordanskii, Yu. B. Lyanda-Geller and G. E. Pikus, JETP Letters 60, 206 (1994); F. G. Pikus and G. E. Pikus, Phys. Rev. B 51, 16928 (1995); A. G. Mal'shukov, K. A. Chao, and M. Willander, Phys. Rev. Lett. 76, 3794 (1996), Physica Scripta T66, 138 (1996).

[14] S. Feng and Y. Hu, Phys. Rev. B 38, 11871 (1988)

[15] B. L. Al'tshuler and B. I. Shklovskii, Sov. Phys. JETP 64, 127 (1986)[Zh. Eksp. Teor. Fiz. 91, 220 (1986)]. 